The Astrophysical Journal, 549:1039-1049, 2001 March 10

(C) 2001. The American Astronomical Society. All rights reserved. Printed in U.S.A.

\title{
THERMAL X-RAY PULSES RESULTING FROM PULSAR GLITCHES
}

\author{
ANisia P. S. TANG AND K. S. ChENG \\ Department of Physics, University of Hong Kong, Pokfulam Road, Hong Kong, China; hrspksc@hkucc.hku.hk \\ Received 1999 July 23 ; accepted 2000 May 6
}

\begin{abstract}
The non-spherically symmetric transport equations and exact thermal evolution model are used to calculate the transient thermal response to pulsar glitches. The three possible forms of energy release from glitches, namely, the "shell," "ring," and "spot" cases, are compared. The X-ray light curves resulting from the thermal response to the glitches are calculated. Only the "spot" case and the "ring" case are considered because the "shell" case does not produce significant modulative X-rays. The magnetic field $(B)$ effect, the relativistic light-bending effect, and the rotational effect on the photons being emitted in a finite region are considered. Different sets of parameters result in different evolution patterns of light curves. We find that this modulated thermal X-ray radiation resulting from glitches may provide some useful constraints on glitch models.
\end{abstract}

Subject headings: dense matter — stars: evolution — stars: interiors — stars: neutron - X-rays: stars

\section{INTRODUCTION}

Pulsar glitches are believed to be mainly either starquake driven or superfluid driven. The starquake mechanism was introduced by Ruderman (1969). He suggested that glitches could be caused by gravity. As a pulsar spins down because of dipole radiation, the centrifugal force on the crust decreases and gravity pulls the crust toward a less oblate shape. Since pulsars are believed to be neutron stars with a solid crust, subsequent change in stellar shape induces stress in the crust until the maximum yield strength is reached. Sudden relaxation of this stress brings the stellar shape to equilibrium. The glitch energy is to be released in a small volume at the weak regions in the solid crust. This leads to the "spot" case as proposed by Van Riper, Epstein, \& Miller (1991). This localized heating process causes an uneven heating of the surface. As the pulsar rotates, the area of thermal photon emission facing the observer is changing. Together with the gravitational bending effect, the emission of thermal X-ray caused by glitches will be at a particular phase in the light curve. Hence a periodic modulation of the $\mathrm{X}$-ray light curve will be observed.

The superfluid-driven glitch mechanism was proposed by Anderson (1975). When the pulsar spins down, the vortices become unpinned from the lattice. They corotate with the local superfluid and scatter off the nearby lattice nuclei. There are two effects. One is superfluid angular momentum being transferred to the crust so that the crust spins up. The other is frictional heating being produced and causing local energy dissipation. Such a process occurs in a ring structure at the rotational equator (Alpar et al. 1984; Cheng et al. 1988). Glitch energy deposition in a ring structure has been studied in detail by other authors (e.g., Bildsten \& Epstein 1989; Epstein \& Baym 1992; Link \& Epstein 1996; Jones 1998).

In reality, the "spotlike" case and the "ringlike" case may not necessarily be mutually exclusive. After crust breaking, which will cause a "spot" case, the whole crust will oscillate briefly about its new equilibrium configuration (Baym \& Pines 1971). The vortices that are originally pinned to the crust will "feel" this oscillation and become unpinned from the crustal lattice. These depinned vortices start to scatter outward and transfer their differential rotational energy to the crust. Therefore, a "ringlike" glitch can follow. As a result, we believe that the energy release in a glitch is mainly composed of two components, namely, the release of elastic energy in a starquake and the differential rotational energy released because of the depinning of vortices. On the other hand, several authors (Van Riper et al. 1991; Chong \& Cheng 1994; Hirano et al. 1997) assume the glitch energy to be released in a spherical shell at a certain density inside the pulsar, namely, the "shell" case, though this does not seem to be realistic. There are other possible origins of the "spot" case of energy release (see, e.g., Srinivasan et al. 1990; Ruderman 1991a; Link, Franco, \& Epstein 1998) and the "ring" case of glitch energy release (e.g., Bildsten \& Epstein 1989; Epstein \& Baym 1992; Jones 1998).

It has been suggested that the transient $\mathrm{X}$-ray emission resulting from glitches will provide a good method to determine the equation of state for pulsars (neutron stars). However, the most active glitching pulsars (e.g., Vela, PSR $1706-16)$ are young neutron stars in which the interior temperature is high $\left(\sim 10^{8} \mathrm{~K}\right)$ - this makes the overall luminosity variation difficult to detect (Van Riper et al. 1991; Chong \& Cheng 1994). Cheng, Li, \& Suen (1998) argue that if a good fraction of glitch energy is released in a small volume, then instead of heating up the entire stellar surface, even a small fraction of glitch energy can heat up a small area of the stellar surface drastically. Therefore, although after a glitch the total X-ray intensity varies very little, a very distinctive hot spot may be detected. They suggest that by observing the transient X-ray pulses, the equations of state of neutron stars can be determined. However, they have not considered such important effects as relativistic light bending (Pechenick, Ftaclas, \& Cohen 1983) and the magnetic field effect (Page 1995), which can significantly affect the intensity and the pulse shape of the transient $\mathrm{X}$-rays resulting from glitches.

In $\S 2$, we summarize the input physics and the relativistic non-spherically symmetric thermal transport and energy balance equations that are used in determining the cooling process following a glitch. In $\S 3$, we apply the scheme mentioned in $\S 2$ to the three cases, namely, the "spot," "ring," and "shell" cases, and compare the temperature and luminosity profiles so obtained. In $\S 4$, we discuss the magnetic field effect, the relativistic light-bending effect, and the rota- 
tional effect. In $\S 5$, we calculate the expected periodic modification to thermal X-ray pulses that are emitted because of "spotlike" and "ringlike" glitches resulting from the effects mentioned in $\S 4$. In $\S 6$, we briefly summarize our results and discuss the detectability of the thermal X-ray profile's being modified because of pulsar glitches.

\section{PHYSICS INPUTS}

No matter what the origins of the glitches are, glitches can be simulated by energy deposition in particular regions in the pulsar. The excess energy propagates in all directions. In this paper, we would like to know what fraction of glitch energy, which produces the transient emission of electromagnetic radiation from the stellar surface, can be observed. In calculating the thermal afterglow effect of glitches, the properties of a neutron star considered to be the major factors affecting the energy flow are the equation of state, the composition, and the initial temperature profile.

\subsection{Neutron Star Structure}

The structure of a neutron star can be constructed from the general relativistic hydrostatic equilibrium equation (the Tolman-Oppenheimer-Volkoff equation),

$$
\frac{d P}{d r}=-\frac{G\left(m+4 \pi r^{2} P / c^{2}\right)\left(\rho+P / c^{2}\right)}{r\left(r-2 G m / c^{2}\right)},
$$

where $P$ and $\rho$ are the pressure and mass density at radius $r$, respectively, and

$$
m=\int_{0}^{r} 4 \pi r^{2} \rho d r
$$

is the gravitational mass inside radius $r . G$ and $c$ are the gravitational constant and the speed of light in a vacuum, respectively.

We will only calculate the temperature profiles between radii with densities from $10^{9} \mathrm{~g} \mathrm{~cm}^{-3}$ to the nuclear density $\rho_{N}$. From $10^{9} \mathrm{~g} \mathrm{~cm}^{-3}$ to the stellar surface, we assume that the region between these two densities reaches equilibrium quickly so that the temperatures at these two densities are related by using the formula proposed by Gudmundsson, Pethick, \& Epstein (1983). In this paper, since different equations of state give rise to different kinds of structure for a neutron star, the major equation of state (EOS) used is UT, which is based on the combined Hamiltonians consisting of $\mathrm{UV}_{14}$ and three-nucleon interaction models (Largaris \& Pandharipande 1981; Friedman \& Pandharipande 1981; Wiringa, Fiks, \& Fabrocini 1988). It is a moderately stiff equation of state when compared with those of the softer BPS model of Baym, Pethick, \& Sutherland (1971) and the stiffer PPS model of Pandharipande, Pines, \& Smith (1976).

\subsection{Composition}

The ion, neutron, and proton mass fractions, electron fraction, and the mass number and proton number in the crust region, denoted by $X_{\text {ion }}, X_{n}, X_{p}, Y_{e}, A$, and $Z$, respectively, are obtained from Lattimer et al. (1985). They are important for calculating the neutrino emissivity, heat capacity, and thermal conductivity as presented in the following subsections.

\subsection{Neutrino Emissivity}

The most important neutrino emission process for pulsars with age $\gtrsim 10^{3} \mathrm{yr}$ is electron bremsstrahlung in the crust (Flowers \& Itoh 1976, 1979), expressed as

$$
Q_{v}^{\text {ions }}=2.1 \times 10^{20} \frac{Z^{2}}{A}\left(\frac{\rho}{\rho_{N}}\right) T_{9}^{6} \mathrm{ergs} \mathrm{cm}^{-3} \mathrm{~s}^{-1},
$$

where $T_{9}$ is the temperature in units of $10^{9} \mathrm{~K}$ and $\rho_{N}=2.8$ $\times 10^{14} \mathrm{~g} \mathrm{~cm}^{-3}$ is the nuclear density. Other neutrino emissivities (cf. Chong \& Cheng 1994 for a brief review) are also included.

\subsection{Heat Capacity $\left(C_{v}\right)$}

In the crust, there are extremely relativistic degenerate electrons, some nonrelativistic neutrons and ions, but there are no free protons. The scheme for calculating heat capacities follows Chong \& Cheng (1994) and Cheng, Li, \& Suen (1998).

\subsection{Thermal Conductivity $(K)$}

Thermal conductivity is fitted according to the work of Itoh et al. (1984) for the solid phase and that of Itoh et al. (1983) for the liquid phase. In regions of $\rho>1.311 \times 10^{14} \mathrm{~g}$ $\mathrm{cm}^{-3}$, linear extrapolation is used.

\subsection{Initial Temperature}

Before a glitch occurs, the temperature of the pulsar should be in equilibrium. For young and middle-aged pulsars, in which glitches can occur, the core temperatures are about $\sim 10^{7}-10^{8} \mathrm{~K}$. Since different EOSs give rise to different kinds of structure, the equilibrium temperature profile of a pulsar depends on the EOS.

\subsection{Non-Spherically Symmetric General Relativistic Transport and Energy Balance Equations}

According to Cheng et al. (1998), the general relativistic thermal transport equation and the energy balance equation are given by

$$
\begin{aligned}
& \frac{e^{-\Phi} e^{-\Lambda}}{r^{2}} \frac{\partial}{\partial r}\left(r^{2} F_{r} e^{2 \Phi}\right)+\frac{e^{\Phi}}{r \sin \theta} \frac{\partial}{\partial \theta}\left(\sin \theta F_{\theta}\right) \\
&+\frac{e^{\Phi}}{r \sin \theta} \frac{\partial}{\partial \phi} F_{\phi}=-\left(C_{v} \frac{d T}{d t}+e^{\Phi} Q_{v}\right) \\
& \frac{\partial}{\partial r}\left(T e^{\Phi}\right)=-\frac{e^{\Phi} e^{\Lambda}}{K} F_{r} \\
& \frac{1}{r} \frac{\partial}{\partial \theta}\left(T e^{\Phi}\right)=-\frac{e^{\Phi}}{K} F_{\theta} \\
& \frac{1}{r \sin \theta} \frac{\partial}{\partial \phi}\left(T e^{\Phi}\right)=-\frac{e^{\Phi}}{K} F_{\phi},
\end{aligned}
$$

where $T$ is the temperature, $e^{\Phi}$ and $e^{\Lambda}$ are the redshift factor and length correction factor, respectively, and $F_{r}, F_{\theta}$, and $F_{\phi}$ are the heat fluxes along the $r$-, $\theta$-, and $\phi$-directions, respectively.

\subsection{Heat Input}

As noted in $\S 1$, there are likely two types of energy release during the glitches, namely, the elastic energy of the crust (Baym \& Pines 1971) and the differential rotation energy between the crustal superfluid and the solid crust. In the former case, the energy is released in a localized volume, 
which is referred to as the "spot" case. In the latter case, the energy is released in the equatorial plane and, hence, is called the "ring" case. The amount of energy released in these two cases is estimated as follows.

\subsection{1. "Spot" Case}

The energy released in a starquake comes from the relief of strain energy and is estimated to be (Baym \& Pines 1971; Ruderman 1991b; Cheng et al. 1992)

$$
\Delta E_{\text {strain }} \sim \mu V_{\text {crust }} \theta_{\max }^{2},
$$

where $\mu\left(\sim 10^{29}\left[\rho /\left(10^{13} \mathrm{~g} \mathrm{~cm}^{-3}\right)\right]^{-4 / 3} \mathrm{dyn} \mathrm{cm}^{-3}\right.$, where $\rho$ is the mass density) is the mean shear modulus, $V_{\text {crust }}$ is the volume of the crust from which energy is released, and $\theta_{\max }$ $\left(\sim 10^{-1}-10^{-2}\right.$ for a pure Coulomb lattice and $10^{-3}-10^{-4}$ for an impurity-dominated lattice; Smolukowski 1970) is the maximum strain angle that the crust can withstand without cracking. If the glitch occurs at $\rho \sim 10^{13} \mathrm{~g} \mathrm{~cm}^{-3}$, the strain energy released is estimated as $\Delta E_{\text {strain }} \sim$ $10^{40}\left(\theta_{\max } / 10^{-2}\right)^{2}$ ergs. Hence, the estimated $\Delta E_{\text {strain }} \lesssim 10^{40}$ ergs.

It has been argued that the magnetic pressure of magnetars, which are neutron stars with extremely strong magnetic fields $\left(\sim 10^{15} \mathrm{G}\right)$, is strong enough to break the crust during the evolution of the magnetic field and may encourage glitching with an amplitude $\Delta \Omega / \Omega \sim 10^{-5}$ (Thompson \& Duncan 1996). In fact, Heyl \& Hernquist (1999) have found evidence for glitches occurring in possible magnetar candidates, 1E 1048.1-5937 and 1E 2259+586, with amplitude $\Delta \Omega / \Omega \sim 10^{-5}$. If a large fraction of magnetic energy is dissipated inside the crust, the heat dissipation resulting from the glitch can be estimated as $I_{*} \Omega^{2}(\Delta \Omega / \Omega) \sim$ $10^{40}$ ergs using the typical parameters of magnetars $(\Omega \sim 1$ $\mathrm{rad} \mathrm{s}^{-1}, \Delta \Omega / \Omega \sim 10^{-5}$, and $I_{*} \sim 10^{45} \mathrm{~g} \mathrm{~cm}^{2}$, where $I_{*}$ is the total moment of inertia of the star).

\subsection{2. "Ring" Case}

In a superfluid-driven glitch originated from a sudden transfer of angular momentum from the inner crust superfluids to the crust, the angular momentum loss for the crustal superfluid is $I_{\mathrm{cr}} \delta \Omega_{s}$, where $I_{\mathrm{cr}}$ is the moment of inertia for the crustal superfluid and $\delta \Omega_{s}$ is the angular velocity change of crustal superfluid before and after the glitch. The angular momentum loss for the charged component, including the stellar core, which is strongly coupled to the solid crust via electron-magnetized vortex scattering (Alpar, Langer, \& Sauls 1984), and the solid crust, is $I_{\mathrm{ch}} \Delta \Omega$, where $\Delta \Omega$ is the observed angular momentum jump of the glitch and $I_{\mathrm{ch}}$ is the moment of inertia for the charged component. By angular momentum conservation, $\Delta J=$ $I_{\mathrm{ch}} \Delta \Omega=I_{\mathrm{cr}} \delta \Omega_{s}$ and the energy dissipated because of the loss of differential rotation energy between the crustal superfluid and the charged component is

$$
\begin{aligned}
\Delta E= & \frac{1}{2}\left[I_{\mathrm{ch}} \Omega^{2}+I_{\mathrm{cr}} \Omega_{s}^{2}\right] \\
& -\frac{1}{2}\left[I_{\mathrm{ch}}(\Omega+\Delta \Omega)^{2}+I_{\mathrm{cr}}\left(\Omega_{s}-\delta \Omega_{s}\right)^{2}\right] \\
= & \Delta J\left(\Omega_{s}-\Omega\right) \equiv \Delta J \Omega_{\mathrm{lag}},
\end{aligned}
$$

where $\Omega_{\text {lag }}$ is the angular speed difference between the crust and the superfluid. In the nuclear pinning region, $\Omega_{\mathrm{lag}}$ is $\sim 1-100 \mathrm{rad} \mathrm{s}^{-1}$ (Alpar, Cheng, \& Pines 1989). In the interstitial pinning region, it is $\lesssim 0.1 \mathrm{rad} \mathrm{s}^{-1}$ (Link \& Epstein 1991). During postglitch relaxation (Epstein, Van Riper, \&
Link 1992; Alpar et al. 1993), $I_{\text {ch }} \gg I_{\text {cr }}$, so $\Delta J=I_{\text {ch }} \Delta \Omega=$ $\left(I_{*}-I_{\mathrm{cr}}\right) \Delta \Omega \simeq I_{*} \Delta \Omega$. For a typical neutron star with a giant glitch, $\Delta \Omega \sim 10^{-4} \mathrm{rad} \mathrm{s}^{-1}$, the moment of inertia of the star is $I_{*} \sim 10^{45} \mathrm{~g} \mathrm{~cm}^{2}$, and the estimated energy release in nuclear pinning regions is $\sim 10^{41}-10^{43} \mathrm{ergs}$, while that in the interstitial pinning regions is $\sim 10^{40}$ ergs.

In general, the starquake glitches (the "spotlike" cases) can occur anywhere within the crust where a Coulomb lattice exists, whereas superfluid-driven glitches (the "ring" cases) can occur only in the inner crust where the neutron superfluid and Coulomb lattices coexist. Therefore, they must occur in the inner crust at densities between $10^{12}$ and $2 \times 10^{14} \mathrm{~g} \mathrm{~cm}^{-3}$. For simplicity, the "ring" case is assumed to occur at $\rho_{\text {glitch }} \sim 10^{13}-10^{14} \mathrm{~g} \mathrm{~cm}^{-3}$. Other cases can occur at $\rho_{\text {glitch }} \sim 10^{12}-10^{14} \mathrm{~g} \mathrm{~cm}^{-3}$. The amount of energy liberated, $\Delta E$, is thus estimated to be between $\sim 10^{40}$ and $10^{43}$ ergs.

\section{COMPARISON OF "SHELL," "RING," AND "SPOT" CASES}

A finite difference method is used to solve the equations of the previous section. For the "shell" case, spherical symmetry can be confidently assumed. As for the "spot" case, the cell at which energy release takes place can be treated as a "pole." The direction joining the center of the pulsar and the "pole" is defined to be the $z$-direction. Since the rotation of the pulsar is slow, the direction of the rotational axis can be neglected and azimuthal symmetry can be assumed. For the "ring" case, the angular velocity direction is taken to be the $z$-direction, so azimuthal symmetry can be assumed again. Therefore, a two-dimensional grid with $N_{r}$ $\times N_{\theta}$ cells is used, where $N_{r}$ and $N_{\theta}$ are, for computational convenience, taken to be 100 and 90 , respectively.

For the "spot" case, the release of energy is in one cell of the grid. For the "shell" case, the release of energy is in a layer of the grid, whereas for the "ring" case the release of energy is around the equator with a height of $4^{\circ}$, i.e., $\sim 0.69$ $\mathrm{km}$ for a UT star, which is roughly the thickness of the crust.

The main parameter used for comparison of these various cases is the total surface luminosity as seen by an observer at infinity, i.e., $L_{s}^{\infty}$. As $L \propto T^{4}$, the increase in temperature is reflected in the luminosity profile.

The temperature profile for a "ring" case glitch is shown in Figure 1. The distribution of surface temperature with respect to the angle from the rotational axis is shown in Figure 2. The distribution of surface temperature with respect to the angle from the spot for the "spot" case is shown in Figure 3. The evolution for the "ring" case is faster than the "spot" case as presented in Cheng et al. (1998), but it is slower than the "shell" case as presented in Chong \& Cheng (1994).

Since the heat propagation time is proportional to the heat capacity, which is approximately proportional to the temperature, i.e., $C_{v} \propto T$, and inversely proportional to the thermal conductivity, which is approximately inversely proportional to the temperature, i.e., $K \propto 1 / T$, the time that the glitch energy requires to reach the stellar surface is roughly proportional to the temperature squared, i.e., $t \propto C_{v} / K \propto T^{2}$ (Hirano et al. 1997). It can be seen that the "shell" case gives the fastest response to the glitch because the same amount of energy is deposited in a larger volume - the localized heating effect is averaged out. Therefore, the temperature in each particular area is not 


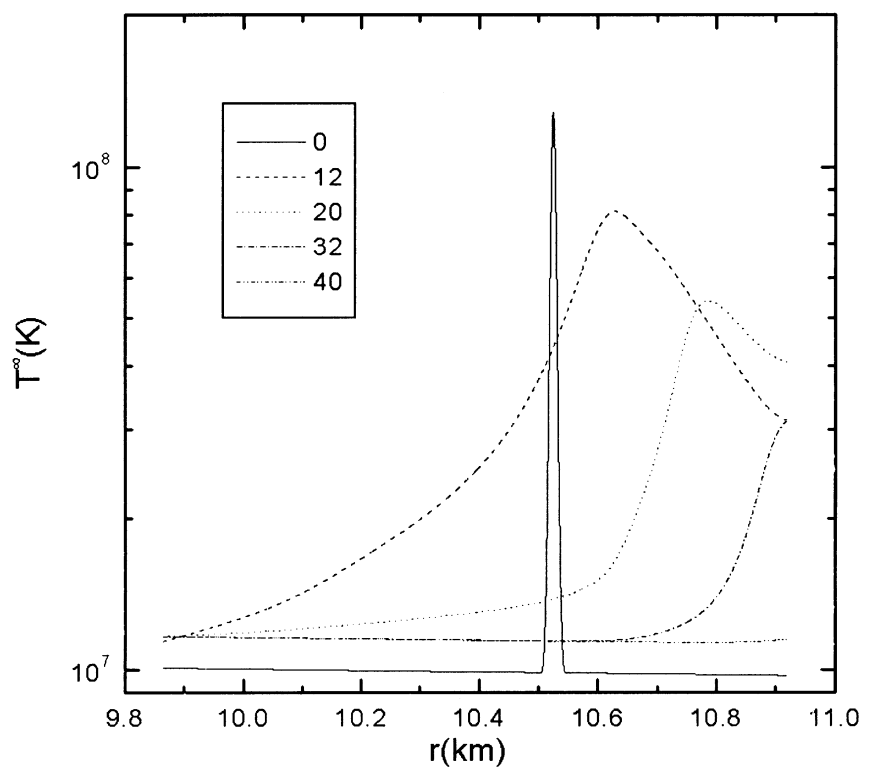

FIG. 1.- Redshifted temperature profile of a UT star with $T_{c}=10^{7} \mathrm{~K}$ and $\Delta E=10^{42}$ ergs at $\rho_{\text {glitch }}=10^{13} \mathrm{~g} \mathrm{~cm}^{-3}$ for the "ring" case with respect to the radius from the center of the star. The key indicates the number of days after the glitch.

so high and the heat energy can reach the surface faster than in the other two cases.

The duration of the pulse on the surface for the "ring" case is also between those for the "shell" case and the "spot" case. This is similar to the "shell" case discussed by Chong \& Cheng (1994), with a larger amount of energy deposited. The pulse will last a longer time because more heat is deposited in a particular volume and this will increase the diffusion time required to the next cell. Therefore, the pulse caused by a "spotlike" glitch will last longer, while the second shortest one is the "ring" case, and, last, the "shell" case.

On the other hand, the peak luminosity for the "spot" case is the highest because the localized heating effect is

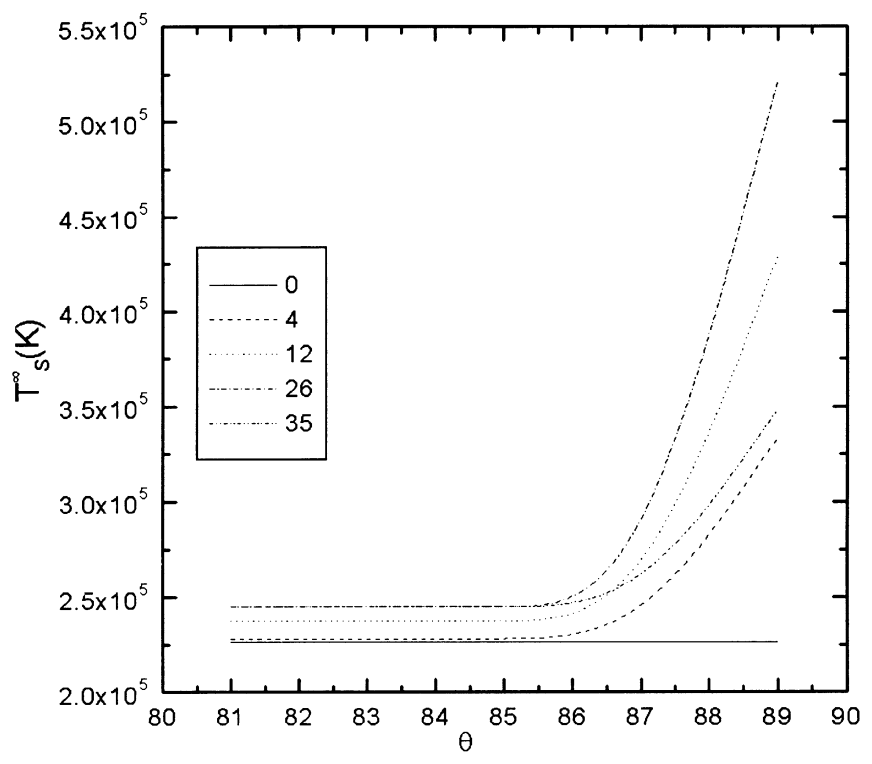

FIG. 2. - Same as Fig. 1, but for redshifted surface temperature

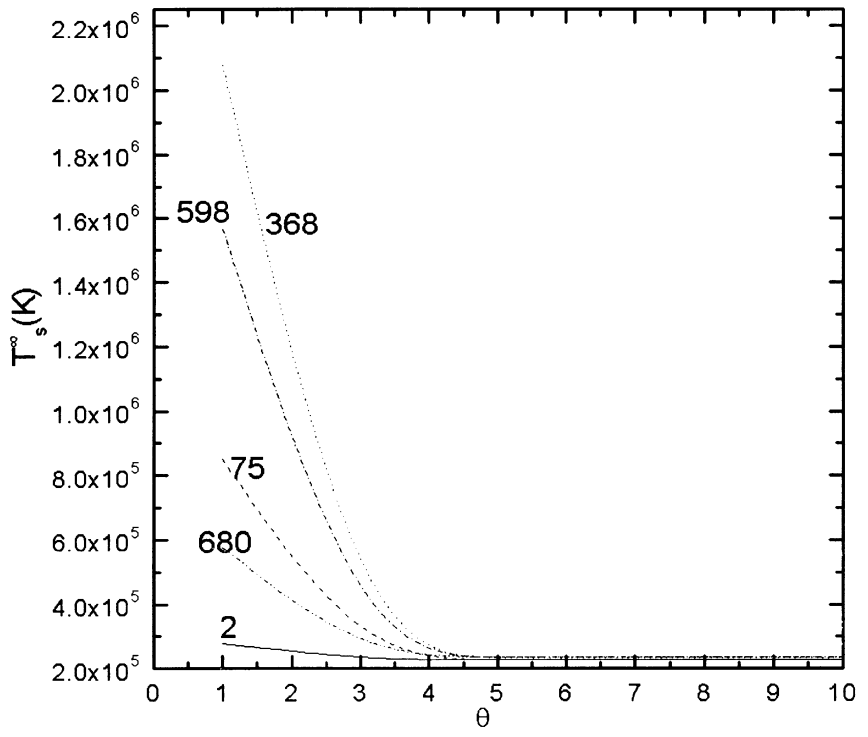

FIG. 3.-Same as Fig. 2, but for the "spot" case. Numbers beside the lines are the number of days after the glitch.

transferred to the surface. Also, the increase in luminosity lasts the longest time for the "spot" case because the heat diffusion speed is the lowest (cf. Figs. 4 and 5). The increase in luminosity is smaller for a cooler core than for a hotter core. The heat content of a pulsar is significantly enhanced by a glitch if the energy liberated is large compared with the original heat content (Chong \& Cheng 1994). This can be understood by noting the fact that luminosity is proportional to $T^{4}$. Hence, the temperature difference is magnified. However, the fractional increase $L_{s}(\max )-L_{s}(0) / L_{s}(0)$ of a cooler core is $\sim 1.3$ and only $\sim 0.014$ for a hotter core. Therefore, it is much easier to observe the transient X-ray pulse from a cooler star than from a hotter star.

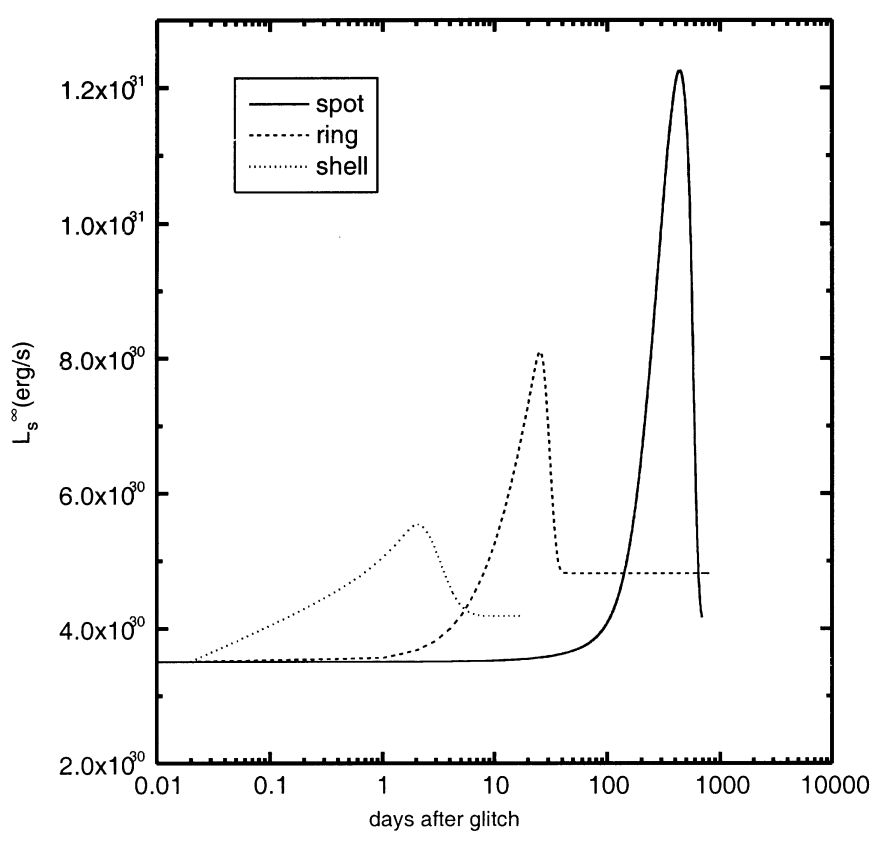

FIG. 4. - Luminosity of a UT star with $T_{c}=10^{7} \mathrm{~K}$ and $\Delta E=10^{42} \mathrm{ergs}$ at $\rho_{\text {glitch }}=10^{13} \mathrm{~g} \mathrm{~cm}^{-3}$. The three cases - "shell," "ring," and "spot"-are compared. 


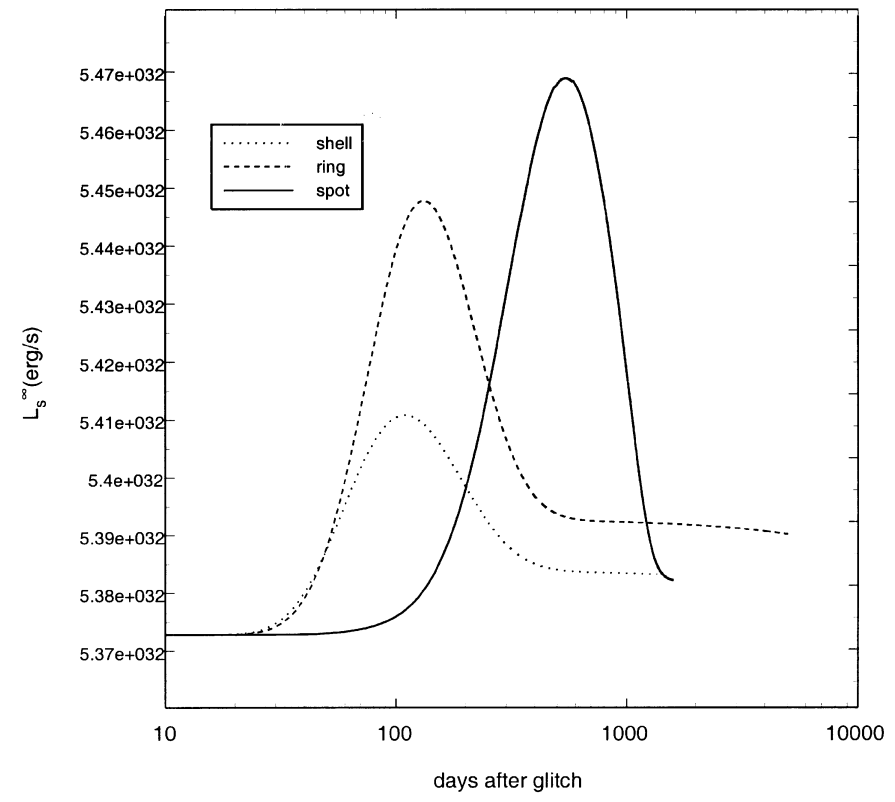

FIG. 5.-Same as Fig. 4, but for $T_{c}=10^{8} \mathrm{~K}$

In Figure 6, we can see that a softer EOS, such as for the BPS star, gives rise to a faster response and a higher peak. This is because a softer EOS gives a thinner crust. Heat diffuses to the surface faster because the distance to the surface is smaller. The deposition of energy to the core will also be smaller, and a larger amount of the released energy is transported to the surface.

\section{THERMAL X-RAY MODIFICATION}

Anderson \& Ögelman (1997) have already proposed that thermal afterglow caused by transient energy releases in a neutron star can alter its X-ray pulse shape by heating a portion of the crust so that more thermal X-rays are emitted at a particular phase. By comparing such kinds of changes in X-ray pulses with the model results, one hopes con-

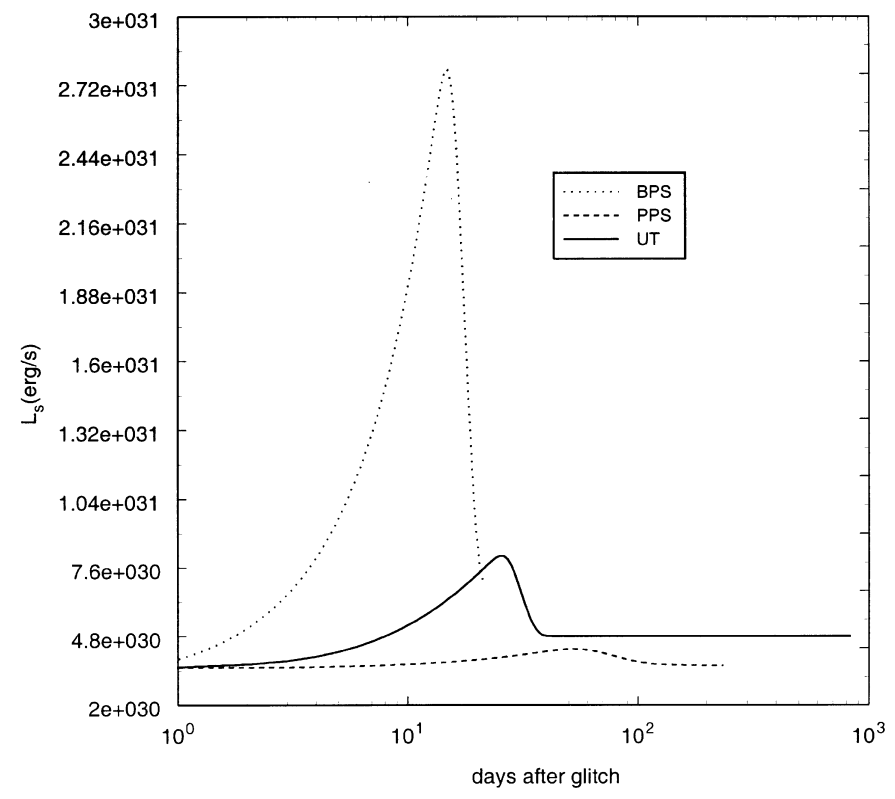

FIG. 6.-Luminosity profiles of a BPS star, a UT star, and a PPS star with $T_{c}=10^{7} \mathrm{~K}$ and $\Delta E=10^{42}$ ergs at $\rho_{\text {glitch }}=10^{13} \mathrm{~g} \mathrm{~cm}^{-3}$ for the "ring" case. straints on glitch models can be made. In the previous section, the temperature profiles on the surface of the pulsar for various models were obtained. However, to calculate thermal X-ray light curves resulting from glitches, the gravitational lensing effect and the magnetic field effect must be considered.

\subsection{Effect of the Magnetic Field}

It is well known that the thermal conductivity in a magnetized neutron star depends on the angle between the heat current and the magnetic field because both the heat conduction and the opacity coefficient depend on the magnetic field direction (Hernquist 1984; Yakovlev \& Urpin 1980; Yakovlev 1982; Tsuruta 1986, 1998; Schaaf 1987, 1988, 1990). The anisotropic conductivity in the crust creates a distribution of the temperature over the stellar surface. Fortunately, it has been shown that an approximate solution to the thermal diffusion equation that accounts for this magnetic field effect can be reduced to a one-dimensional problem and the surface temperature distribution is given by (e.g., Page 1995)

$$
\begin{aligned}
T_{s, \text { ani }}^{4}\left(\boldsymbol{B}, T_{b}, \Theta_{B}\right)= & T_{s, \text { iso }}^{4}\left(\boldsymbol{B}=0, T_{b}, \Theta_{B}=0\right) \\
& \times\left[\cos ^{2} \Theta_{B}+\chi_{0}^{4}\left(\boldsymbol{B}, T_{b}\right) \sin ^{2} \Theta_{B}\right]
\end{aligned}
$$

with $\chi_{0}^{4}=K_{\perp} / K_{\|}$, the ratio of the thermal conductivities perpendicular and parallel to the magnetic field, which in accordance with Greenstein \& Hartke (1983) is assumed to be constant within the envelope. Page (1995) further establishes $\chi_{0}$ as $T_{s, \text { ani }}\left(\Theta_{B}=90^{\circ}\right) / T_{s \text {, ani }}\left(\Theta_{B}=0^{\circ}\right)$ in. $\Theta_{B}$ is the angle between the local field and the radial direction. Here, only the dipolar magnetic field case is considered. $T_{b}$ is the temperature at a density of $10^{10} \mathrm{~g} \mathrm{~cm}^{-3}, T_{s, \text { ani }}$ is the surface temperature of the anisotropic case, and $T_{s, \text { iso }}$ is the surface temperature of the isotropic (no magnetic field) case.

\subsection{The Effect of Relativistic Light Bending}

Harding \& Muslimov (1998) modeled the soft thermal X-ray profile by noting that the magnetic polar caps of a cooling neutron star are slightly hotter than the rest of the stellar surface because of the strong magnetic field effect on heat transport in the surface layers. A similar approach is used to analyze the "spot" case in this paper, but the origin of the excessive heating is different. In fact, in their case, a steady X-ray pulse is expected, but in our case, a transient X-ray pulse occurs. In this paper, the effect of the magnetic field is ignored.

The following scheme of calculating the X-ray light curves resulting from gravitational bending and the stellar rotation is adapted from Pechenick et al. (1983). Different model temperature profiles of the "spot" case are incorporated to determine the energy flux emitted from different positions of the pulsar. Since a neutron star has a large mass and a small radius, it is necessary to consider the gravitational deflection of the emitted photons (cf. Fig. 7). When the photons are emitted at an angle $\delta$ from the observer's direction, it will seem to the observer that they are emitted at an angle $\theta^{\prime}$. The relationship between $\delta$ and $\theta^{\prime}$ can be generated from the equations given by Pechenick et al. (1983):

$$
\begin{gathered}
\xi+\beta=\theta^{\prime} \\
\beta=\int_{0}^{G M / R c^{2}}\left[\left(\frac{D \xi c^{2}}{G M}\right)^{-2}-(1-2 u) u^{2}\right]^{-1 / 2} d u,
\end{gathered}
$$




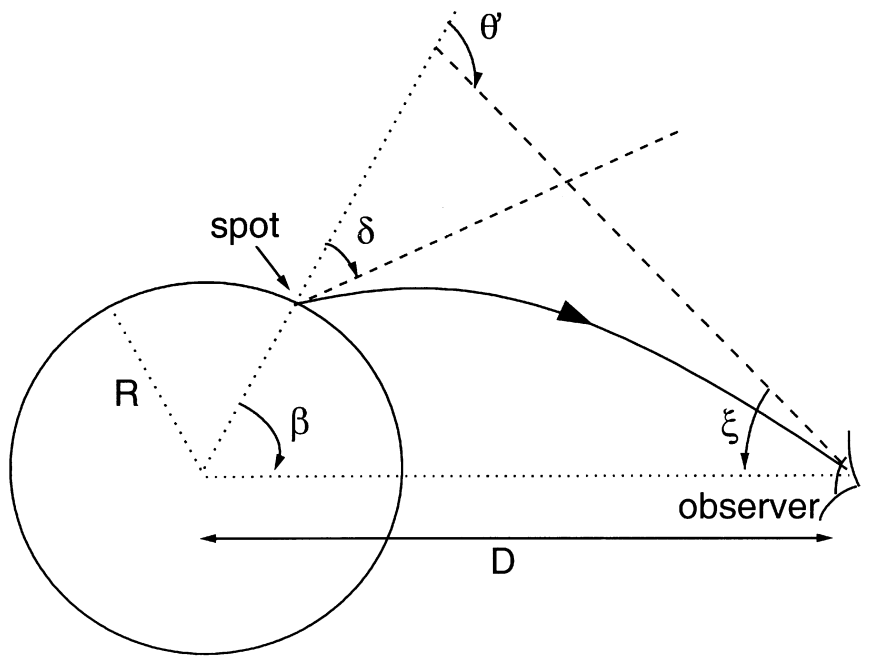

FIG. 7.-Schematic illustration of gravitational lensing effect. Angles are defined as shown.

$$
\delta=\sin ^{-1}\left(\frac{D \sin \xi}{R} \sqrt{\frac{1-2 G M / R c^{2}}{1-2 G M / D c^{2}}}\right),
$$

where $D$ is the distance to the observer and the angles are defined as in Figure 7. The numerical results for the relationship between $\delta$ and $\theta^{\prime}$ for different EOSs can be found in Figure 3 of Page (1995), which shows that softer equations of state give rise to stronger lensing effects.

To avoid the complex procedures in solving the above three equations, some approximations can be made. Since $2 G M / D c^{2}$ is extremely small, and $\xi$ is also very small, $\delta$ can be treated as $(D \xi / R)\left(1-G M / R c^{2}\right)$ and $\beta \approx$ $\int_{0}^{G M / R c^{2}}\left(D \xi c^{2} / G M\right)\left(1+\frac{1}{2} u^{2}\left[D \xi /\left(G M / c^{2}\right)\right]^{2}\right) d u$, which is approximately $D \xi / R+\frac{1}{6}\left(G M / R c^{2}\right)^{3}\left[D \xi /\left(G M / c^{2}\right)\right]^{3}=D \xi / R$ $+\frac{1}{6}(D \xi / R)^{3}$. As a result, $\theta^{\prime} \approx \beta \approx(D / R) \xi+\frac{1}{6}(D \xi / R)^{3}$. Hence, $\theta^{\prime}-\delta \approx\left(G M / R c^{2}\right) \delta$. Actually, this is a good approximation for $\delta<60^{\circ}$.

Since the rotation speed of a pulsar is much less than the speed of light, it can be treated as a slowly rotating rigid body, in which case

$$
\cos \theta_{0}=\sin \gamma \sin \gamma_{o} \cos \Omega t+\cos \gamma \cos \gamma_{o},
$$

where $\theta_{0}$ is the angle between the center of the "spot" region and the observer and $\gamma$ and $\gamma_{o}$ are the position angles from the $z$-axis to the center of the "spot" region and the observer, respectively, as described in Figure $8 . \Omega t$ is the azimuthal angle at time $t$, and $\Omega$ is the stellar angular velocity. So far, we have considered the emission region as a point; however, in reality, the emission region is of a finite area. If the photon emission occurs at a surface area defined in a cone with an angle $\alpha$ at the stellar surface, the result is then generated from the following equations according to Pechenick et al. (1983):

$$
h\left(\theta ; \alpha, \theta_{0}\right)=2 \cos ^{-1}\left(\frac{\cos \alpha-\cos \theta_{0} \cos \theta}{\sin \theta_{0} \sin \theta}\right)
$$

for $\theta$ in the range $\theta_{0} \pm$ and where

$$
\theta(x)=\int_{0}^{G M / R c^{2}}\left[x^{-2}-(1-2 u) u^{2}\right]^{-1 / 2} d u ;
$$

$\theta_{0}$ is defined in the same way as in equation (14). The rela-

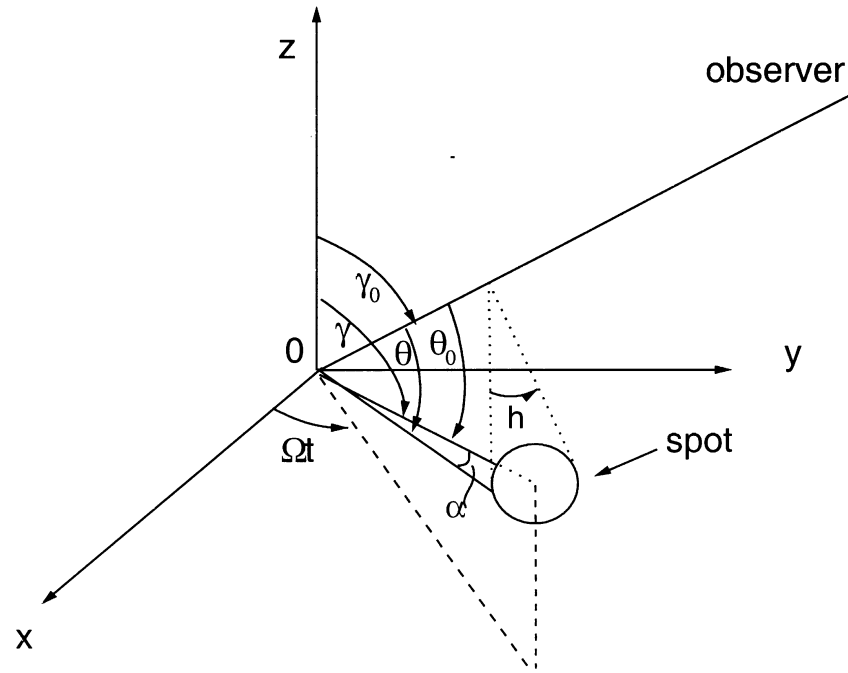

FIG. 8.-Angles used in calculating the rotational effect

tive brightness is

$$
\begin{aligned}
A\left(\theta_{0} ; f, M / R, \alpha\right)=(1 & \left.-2 G M / R c^{2}\right)^{2}\left(G M / R c^{2}\right)^{2} \\
& \times \int_{0}^{x_{\max }} f(\delta(x)) h\left(x ; \alpha, \theta_{0}\right) x d x,
\end{aligned}
$$

where $f(\delta)=1$ for isotropic emission, $f(\delta)=\cos \delta$ for enhanced emission, and $f(\delta)=\sin \delta$ for suppressed emission, $\delta=\sin ^{-1}\left(x / x_{\text {max }}\right)$. As $\xi$ is small, $\sin \xi$ is approximated as $\xi$, so $x=D \xi c^{2} / G M$ and

$$
x_{\max }=\left(R c^{2} / G M\right)\left(1-2 G M / R c^{2}\right)^{-1 / 2},
$$

where the angles are as defined in Figure 8.

The relationship between $A$ and $\Omega t$ is plotted in Figure 9 for isotropic emission. Our results are similar to Figure 9 of Page (1995) and Figure 7 of Heyl \& Hernquist (1998). Generally, a softer EOS gives a stronger lensing effect so that

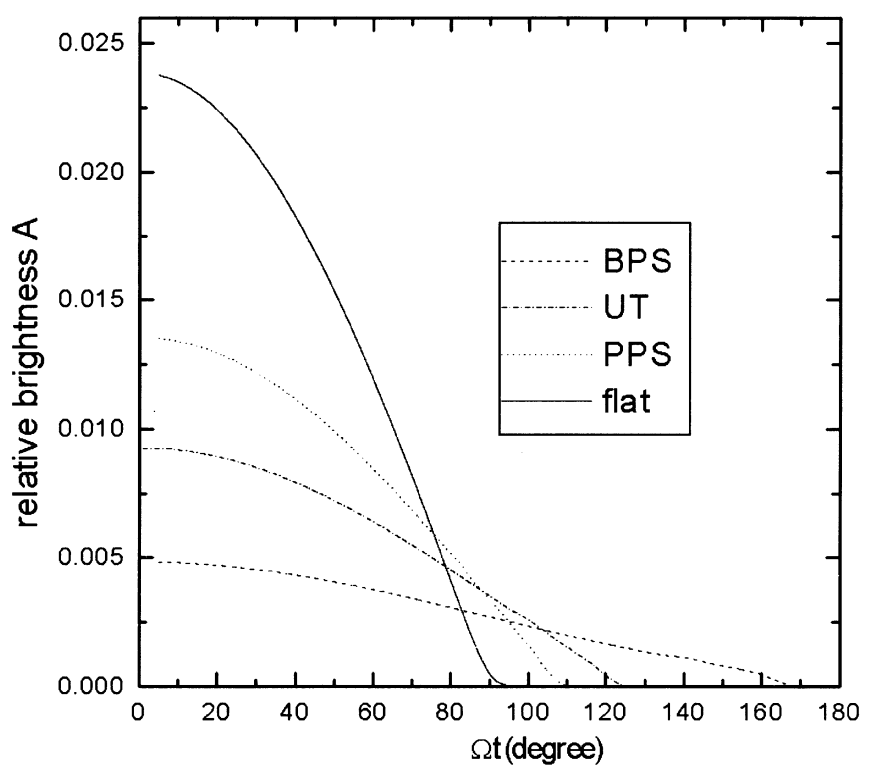

FIG. 9.-Finite area for the "spot" considered for different EOSs: BPS, PPS, and UT. Angular radius of the emission cone is $\alpha=5^{\circ}$. 


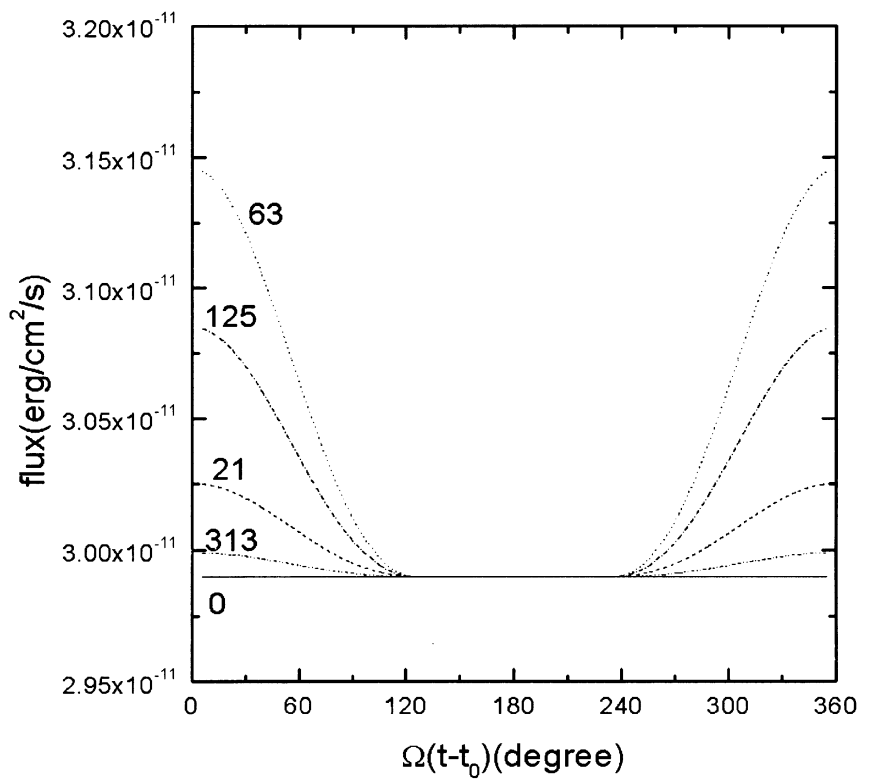

FIG. 10.-Evolution of the total brightness for a UT star with $T_{c}=10^{8}$ $\mathrm{K}$ at $\rho_{\text {glitch }}=10^{11} \mathrm{~g} \mathrm{~cm}^{-3}$ with $\Delta E=10^{40}$ ergs for the "spot" case.

when the "spot" is nearly at the back $\left(\sim 170^{\circ}\right)$, it can still be observed even though the relative brightness is low. For a UT star, the bending effect can extend the observed angle to nearly $30^{\circ}$. With a larger cone of emission, the relative brightness is larger when the "spot" is facing the observer, but it does not have any effect on the maximum angle of deflection.

According to Pechenick et al. (1983), the total energy flux observed is

$$
F_{\mathrm{X}}=\sum I_{0}\left(\frac{R}{D}\right)^{2} A\left(\theta_{0} ; f, M / R, \alpha\right),
$$

where $I_{0}$ is the energy flux at the surface for different angles from the spin axis and includes the factors $\cos \delta$, and the summation is over the contributions from each cell.

Including the gravitational bending, together with the slow rotation and the finite-area effects, the evolution of the total brightness for a UT star with $T_{c}=10^{8} \mathrm{~K}, \Delta E=$ $10^{40}$ ergs, and $\rho_{\text {glitch }}=10^{11} \mathrm{~g} \mathrm{~cm}^{-3}$ is plotted in Figure 10. Other sets of parameters give similarly shaped light curves, except that the number of days to reach the maximum will change, as will the fractional increase in energy flux.

\section{NUMERICAL RESULTS}

Consider the fractions $f_{F}=\left(F_{\mathrm{X}_{\max }}-F_{\mathrm{X}_{\text {min }}}\right) / F_{\mathrm{X}_{\min }}$ and $f_{T}=\left(T_{s_{\max }}-T_{s_{\min }}\right) / T_{s_{\min }}$, which measure the visibility of the X-ray pulses caused by a "spotlike" glitch in one particular rotation around the peak of the luminosity-time graphs. The numerical values are given in Table 1, where the magnetic field effect has not yet been included.

If $f_{F} \geq 0.05$, it should be large enough for observation, and then the cases with $T_{c}=10^{7} \mathrm{~K}, \Delta E=10^{39} \mathrm{ergs}$, and $\rho_{\text {glitch }}=10^{11} \mathrm{~g} \mathrm{~cm}^{-3} ; T_{c}=10^{8} \mathrm{~K}, \Delta E=10^{39}$ ergs, and $\rho_{\text {glitch }}=10^{10} \mathrm{~g} \mathrm{~cm}{ }^{-3} ; T_{c}=10^{8} \mathrm{~K}, \Delta E=10^{40}$ ergs, and $\rho_{\text {glitch }}=10^{11} \mathrm{~g} \mathrm{~cm}^{-3}$; and $T_{c}=10^{7} \mathrm{~K}, \Delta E=10^{42}$ ergs, and $\rho_{\text {glitch }}=10^{13} \mathrm{~g} \mathrm{~cm}^{-3}$ will be possible to observe.

In the key to Figure 11, the first number is the angle of the "spot" from the rotational axis and the second number
TABLE 1

Numerical Results for the "Spot" CASE of UT EQUATION OF STATE WITH No B-FIELD

\begin{tabular}{|c|c|c|c|}
\hline $\begin{array}{c}\rho_{\text {glitch }} \\
\left(\mathrm{g} \mathrm{cm}^{-3}\right)\end{array}$ & $\begin{array}{c}\Delta E \\
\text { (ergs) }\end{array}$ & $f_{F}$ & $f_{T}$ \\
\hline \multicolumn{4}{|l|}{$T_{\text {core }} 10^{8} \mathrm{~K}:$} \\
\hline \multirow{5}{*}{$10^{11} \ldots \ldots \ldots .}$. & $10^{38}$ & 0.0002 & 0.0395 \\
\hline & $10^{39}$ & 0.0033 & 0.3400 \\
\hline & $10^{40}$ & 0.0517 & 1.4493 \\
\hline & $10^{41}$ & 1.8673 & 4.9635 \\
\hline & $10^{42}$ & 50.8427 & 12.6201 \\
\hline \multirow[t]{2}{*}{$10^{10} \ldots \ldots \ldots$} & $10^{38}$ & 0.0033 & 0.3411 \\
\hline & $10^{39}$ & 0.0814 & 1.7365 \\
\hline $3 \times 10^{11}$. & $10^{40}$ & 0.0097 & 0.6568 \\
\hline $10^{13}$ & $10^{42}$ & 0.0776 & 1.7047 \\
\hline \multicolumn{4}{|l|}{$T_{\text {core }} 10^{7} \mathrm{~K}:$} \\
\hline \multirow[t]{2}{*}{$\begin{array}{c}\text { core } \\
10^{11} \ldots \ldots \ldots \ldots . . .\end{array}$} & $10^{38}$ & 0.0183 & 0.9121 \\
\hline & $10^{39}$ & 0.3670 & 3.8872 \\
\hline $10^{12}$ & $10^{42}$ & 66.2741 & 13.5537 \\
\hline $10^{13} \ldots$ & $10^{42}$ & 9.0100 & 7.8376 \\
\hline
\end{tabular}

NoTE.-The fractions $f_{F}$ and $f_{T}$ are calculated for various sets of parameters with the "spot" case of UT equation of state with no $B$ field and spot and the observer both lying along the rotational equator.

is the angle of the observer from the rotational axis. Other numerical results can be found in Tang (1999).

Taking the magnetic field effect into consideration, the background light curve is no longer a straight line. That is to say, the two magnetic poles have produced modulation of the flux profiles already. For easy calculation, the background light curves in the figures in this second part of the section are generated by using a $10^{\circ} \times 10^{\circ}$ grid; the rotational axis $\boldsymbol{\Omega}$ is taken to be the $z$-axis; the magnetic moment $\mu$ is taken to be at an angle of $45^{\circ}$ from $\Omega$; the "spot" case glitch has its energy released at an angle of $60^{\circ}$ from $\Omega$ and $90^{\circ}$ azimuthally from $\mu$. In other words, if $(\Omega, \mu)$ is the $z-y$ plane, then the spot is in the $z-x$ plane. The "ring" case glitch has its energy released along the rotational equator. The observer is at an angle of $45^{\circ}$ from $\Omega$.

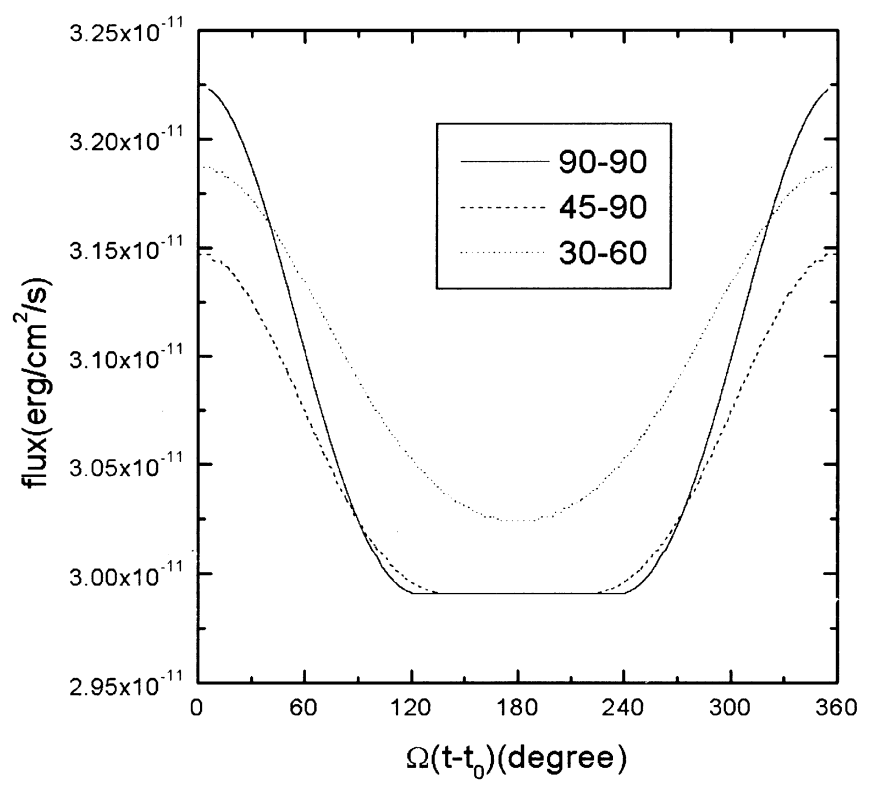

FIG. 11.- Total brightness for a UT star with $T_{c}=10^{8} \mathrm{~K}$ at $\rho_{\text {glitch }}=$ $10^{13} \mathrm{~g} \mathrm{~cm}^{-3}$ with $\Delta E=10^{42}$ ergs 418 days after a "spotlike" glitch. 
For a "spotlike" glitch, the glitch effect alone can produce modulation to the X-ray profiles, as mentioned in the beginning of this section. It can significantly increase the fraction $f_{F}$ with some particular sets of parameters. If there is a magnetic field effect, it can also cause a phase shift of the whole light curve, as seen in Figures 12, 13, 14, and 15. The results are summarized in Table 2.

For the "ring" case, if there is no magnetic field, there will be no modulation to the light curve caused by azimuthal symmetry. Only the observed total flux is increased. If a magnetic field effect is also considered, the glitch effect causes the peak of the X-ray profile to rise and the pulse shape to shift. However, it depresses the contrast $f_{F}$. This is easily observed from the values in Table 3 and in Figures 16,17 , and 18 .

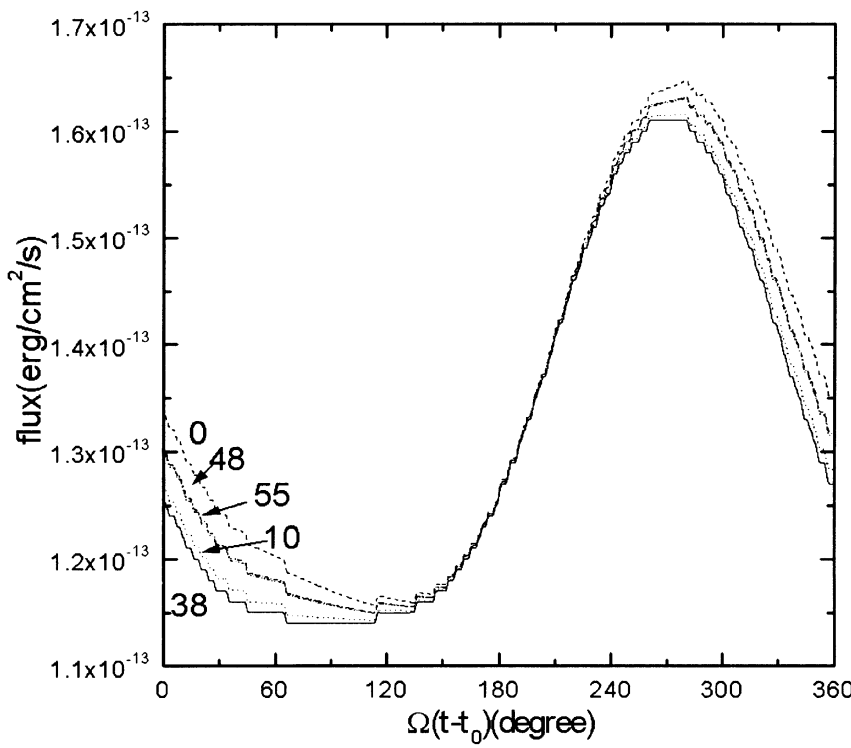

FIG. 12. - Evolution of the total brightness for a UT star with $T_{c}=10^{7}$ $\mathrm{K}$ at $\rho_{\mathrm{glitch}}=10^{12} \mathrm{~g} \mathrm{~cm}^{-3}$ with $\Delta E=10^{40}$ ergs for the "spot" case with magnetic field $B=3 \times 10^{12} \mathrm{G}$.

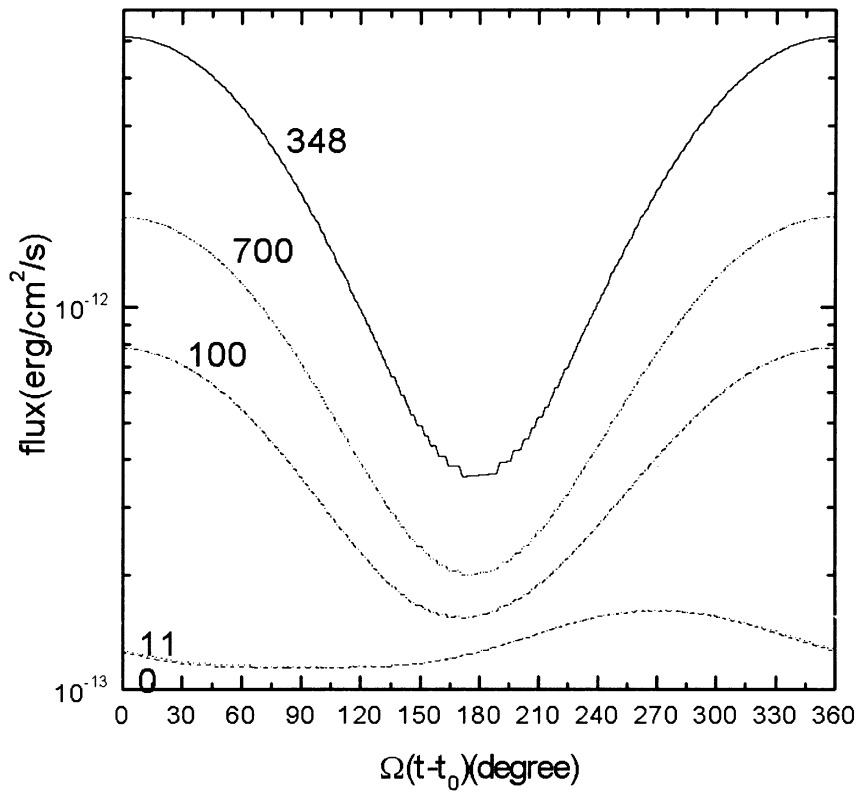

FIG. 13.- Same as Fig. 12, but for $\Delta E=10^{42}$ ergs

The rise of the peak flux value can be more drastic for the "spot" case than the "ring" case with the same set of parameters, especially at low temperature, e.g., $T_{\text {core }}=10^{7}$ $\mathrm{K}$, and with a large amount of energy deposition, e.g., $\Delta E=10^{42}$ ergs. The amount of phase shift of the light curve is determined by the position at which the glitch energy is released (its azimuthal angular distance from $\mu$ ). For the "spot" case, since we choose the "spot" to be $90^{\circ}$ from $\mu$ azimuthally, there is a $90^{\circ}$ phase shift in the X-ray light curve when the glitch energy reaches the surface (cf. Fig. 13). For the "ring" case, the additional peak results from the glitch (cf. Fig. 17). For both the "spot" and the "ring" cases, the values of $f_{F}$ are greater than 0.05 . As a result, we expect that the change in the light curve due to the glitch should be observable. In fact, the changes in pulse shape point to the corresponding sets of parameters.

TABLE 2

Numerical Results for the “Spot” Case of UT Equation of State with $\boldsymbol{B}$ at $45^{\circ}$ From $\Omega$

\begin{tabular}{|c|c|c|c|c|c|c|}
\hline $\begin{array}{l}T_{\text {core }} \\
(\mathrm{K})\end{array}$ & $\begin{array}{c}\rho_{\text {glitch }} \\
\left(\mathrm{g} \mathrm{cm}^{-3}\right)\end{array}$ & $\begin{array}{c}E_{\gamma} \\
\text { (ergs) }\end{array}$ & $\begin{array}{c}B \\
(\mathrm{G})\end{array}$ & $f_{F}$ before Glitch & $f_{F}$ at $t_{\max }$ & New Peak to Old Peak \\
\hline \multicolumn{7}{|c|}{$\Delta E=10^{40}$ ergs: } \\
\hline \multirow[t]{3}{*}{$10^{7} \ldots \ldots$} & $10^{12}$ & $3.97 \times 10^{35}$ & 0 & 0.00 & 0.09 & 1.09 \\
\hline & & & $3 \times 10^{12}$ & 0.41 & 0.42 & 1.02 \\
\hline & & & $10^{15}$ & 0.41 & 0.41 & 1.00 \\
\hline \multirow[t]{3}{*}{$10^{8} \ldots \ldots$} & $10^{12}$ & $5.07 \times 10^{37}$ & 0 & 0.00 & 0.00 & 1.00 \\
\hline & & & $3 \times 10^{12}$ & 0.41 & 0.41 & 1.00 \\
\hline & & & $10^{15}$ & 0.40 & 0.40 & 1.00 \\
\hline \multicolumn{7}{|c|}{$\Delta E=10^{42}$ ergs: } \\
\hline \multirow[t]{3}{*}{$10^{7} \ldots \ldots$} & $10^{12}$ & $1.91 \times 10^{39}$ & 0 & 0.00 & 14.52 & 56.76 \\
\hline & & & $3 \times 10^{12}$ & 0.41 & 13.20 & 31.86 \\
\hline & & & $10^{15}$ & 0.41 & 13.23 & 31.78 \\
\hline \multirow[t]{3}{*}{$10^{8} \ldots \ldots$} & $10^{12}$ & $1.91 \times 10^{39}$ & 0 & 0.00 & 0.34 & 1.36 \\
\hline & & & $3 \times 10^{12}$ & 0.41 & 0.49 & 1.10 \\
\hline & & & $10^{15}$ & 0.40 & 0.49 & 1.10 \\
\hline
\end{tabular}

NOTE.-The fractions $f_{F}$ are calculated for different sets of parameters for the "spot" case of the UT equation of state with $B$ at $45^{\circ}$ from $\Omega$, the "spot" located at an angle of $60^{\circ}$ from $\Omega$, and $90^{\circ}$ azimuthally from $B$, and with the observer at an angle $45^{\circ}$ with $\Omega$. The "new peak" represents the maximum flux value after the glitch, and the "old peak" represents the value before the glitch. The value $E_{\gamma}=\int_{0}^{2 t_{\max }}\left[L(t)-L_{\mathrm{BG}}\right] d t$ is the amount of energy that is emitted as photons at the surface; $t_{\max }$ is the time at which the luminosity has its peak value after the glitch; and $L_{\mathbf{B G}}$ is the background luminosity. 


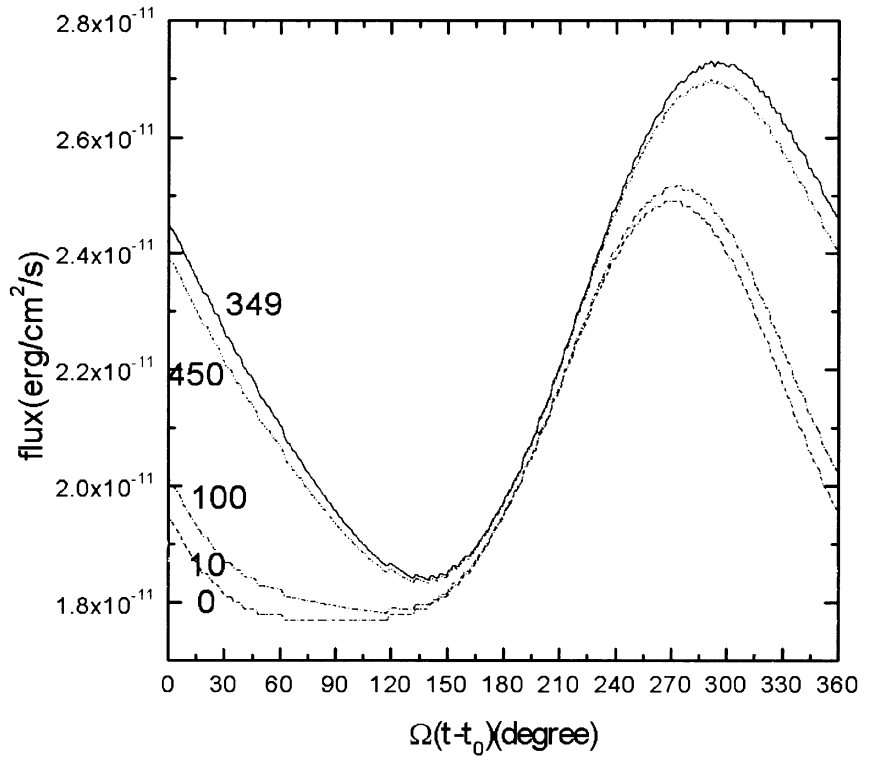

FIG. 14. - Same as Fig. 13, but for $T_{c}=10^{8} \mathrm{~K}$

It is important to note that with the magnetic field effect, heat transport becomes a three-dimensional problem even for the "ring" case. The energy released in a glitch can have two routes. Part of it is transferred to the core (cf. Fig. 1). The core is able to quickly regain its isothermality, and the energy is reradiated isotropically to both directions of the magnetic pole and the magnetic equator. According to Harding \& Muslimov (1998), the transverse heat conductivity is suppressed because of magnetization of electrons, which results in a surface temperature at the magnetic pole higher than at the equator. The other part of the glitch energy is transferred to the crust since the heat propagation time is proportional to the temperature squared, as mentioned in $\S 3$. The distance from and the temperature of the heated area are the main elements affecting the angular distribution of the thermal energy on the surface.

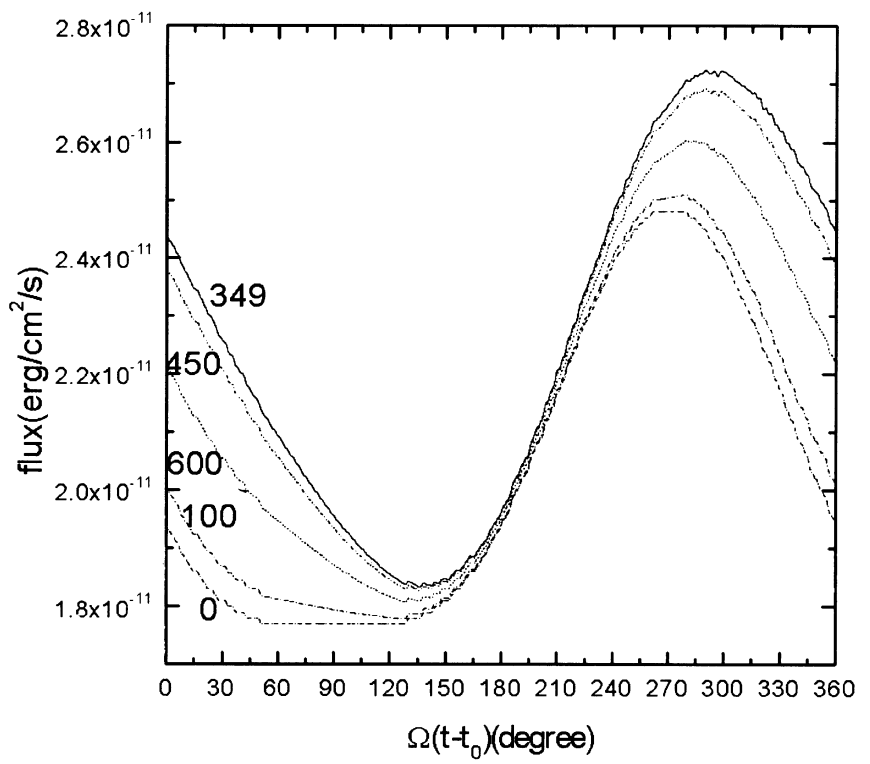

FIG. 15.-Same as Fig. 14, but for $B=10^{15} \mathrm{G}$

When the "ring" case is compared with the "spot" case, the same amount of energy is deposited in a larger volume and the localized heating effect is averaged out. Therefore, the temperature in each particular volume element is not so high. Hence, the heat energy can reach the surface faster. Comparing Figure 1, which describes the thermal evolution of a "ringlike" glitch, with Figure 1 in Cheng \& Li (1997), which describes the thermal evolution of a "spotlike" glitch, this effect can be seen easily. That means, for a "ringlike" glitch, a larger part of the glitch energy is transferred to the crust than to the core. Since the magnetic pole and the magnetic equator are at the same distance from the "ring" (with the magnetic moment $\mu$ at an angle of $45^{\circ}$ from $\boldsymbol{\Omega}$ and the "ring" $90^{\circ}$ from $\boldsymbol{\Omega}$ ), and the magnetic pole is hotter, energy transport in the magnetic equator direction is faster-hence decreasing the contrast $f_{F}$, as shown in

TABLE 3

Numerical Results for Various Sets of Parameters for the “Ring” Case of UT Equation of State

\begin{tabular}{|c|c|c|c|c|c|c|}
\hline $\begin{array}{l}T_{\text {core }} \\
(\mathrm{K})\end{array}$ & $\begin{array}{c}\rho_{\mathrm{glitch}} \\
\left(\mathrm{g} \mathrm{cm}^{-3}\right)\end{array}$ & $\begin{array}{c}E_{\gamma} \\
\text { (ergs) }\end{array}$ & $\begin{array}{c}\boldsymbol{B} \\
(\mathrm{G})\end{array}$ & $f_{F}$ before Glitch & $f_{F}$ at $t_{\max }$ & New Peak to Old Peak \\
\hline \multicolumn{7}{|c|}{$\Delta E=10^{40}$ ergs: } \\
\hline \multirow[t]{3}{*}{$10^{7} \ldots \ldots$} & $10^{12}$ & $2.00 \times 10^{35}$ & 0 & 0.00 & 0.00 & 1.09 \\
\hline & & & $3 \times 10^{12}$ & 0.41 & 0.38 & 1.07 \\
\hline & & & $10^{15}$ & 0.41 & 0.38 & 1.07 \\
\hline \multirow[t]{3}{*}{$10^{8} \ldots \ldots$} & $10^{12}$ & $1.68 \times 10^{35}$ & 0 & 0.00 & 0.00 & 1.00 \\
\hline & & & $3 \times 10^{12}$ & 0.40 & 0.41 & 1.00 \\
\hline & & & $10^{15}$ & 0.41 & 0.40 & 1.00 \\
\hline \multicolumn{7}{|c|}{$\Delta E=10^{42}$ ergs: } \\
\hline \multirow[t]{3}{*}{$10^{7} \ldots \ldots$} & $10^{12}$ & $9.80 \times 10^{37}$ & 0 & 0.00 & 0.00 & 11.61 \\
\hline & & & $3 \times 10^{12}$ & 0.41 & 0.20 & 9.66 \\
\hline & & & $10^{15}$ & 0.41 & 0.20 & 9.65 \\
\hline \multirow[t]{3}{*}{$10^{8} \ldots \ldots$} & $10^{12}$ & $1.02 \times 10^{38}$ & 0 & 0.00 & 0.00 & 1.07 \\
\hline & & & $3 \times 10^{12}$ & 0.40 & 0.38 & 1.06 \\
\hline & & & $10^{15}$ & 0.41 & 0.38 & 1.06 \\
\hline
\end{tabular}

NOTE.-The fractions $f_{F}$ are calculated for different sets of parameters for the "ring" case of the UT equation of state with $\mu$, as well as the observer, making an angle of $45^{\circ}$ with $\Omega$. The "new peak" represents the maximum flux value after the glitch and the "old peak" represents this value before the glitch. The value $E_{\gamma}=\int_{0}^{2 t_{\max }}\left[L(t)-L_{\mathbf{B G}}\right] d t$ is the amount of energy emitted as photons at the surface; $t_{\max }$ is the time at which the luminosity has its peak value after the glitch; and $L_{\mathbf{B G}}$ is the background luminosity. 


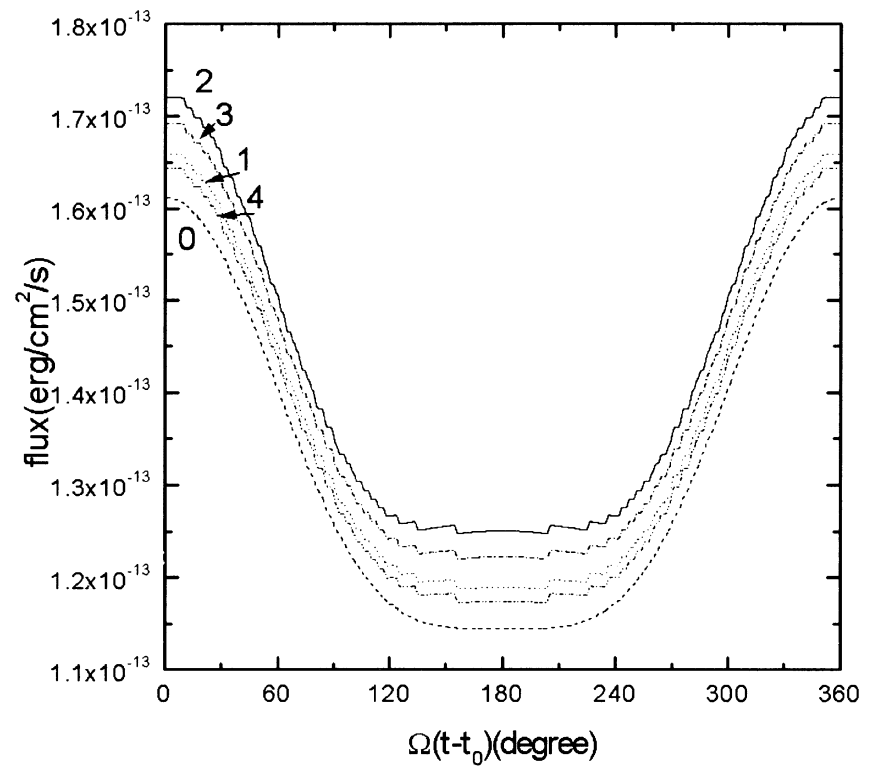

FIG. 16.-Evolution of the total brightness for a UT star with $T_{c}=10^{7}$ $\mathrm{K}$ at $\rho_{\text {glitch }}=10^{12} \mathrm{~g} \mathrm{~cm}^{-3}$ with $\Delta E=10^{40} \mathrm{ergs}$ for the "ring" case with magnetic field $B=3 \times 10^{12} \mathrm{G}$.

Table 3. For the "spot" case, however, a larger fraction of the glitch energy is transported to the core, which explains the increase in the contrast $f_{F}$ for the "spot" case.

For the case of low temperature, $T_{\text {core }}=10^{7} \mathrm{~K}$, and large energy deposition, $\Delta E=10^{42}$ ergs, since the localized heating is enormous, the effect of polar cap heating can be viewed simply as a modification to the uniform temperature profile. The magnetic effect is absolutely overwhelmed by the glitch effect. However, for a young star such as Vela, the core temperature is still high $\left(\sim 10^{8} \mathrm{~K}\right)$, the rate of energy transport is slower, and hence a larger fraction of the glitch energy is transferred to the core. Therefore, the glitch effect is not significant in terms of both phase shift and the rise of the peak flux.

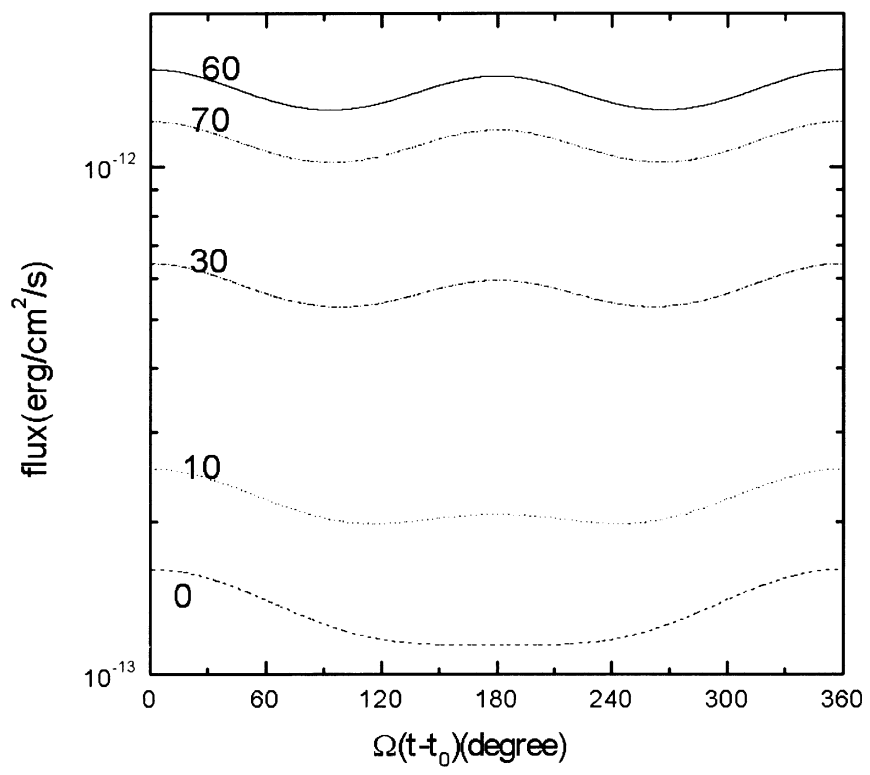

FIG. 17. - Same as Fig. 16, but for $\Delta E=10^{42}$

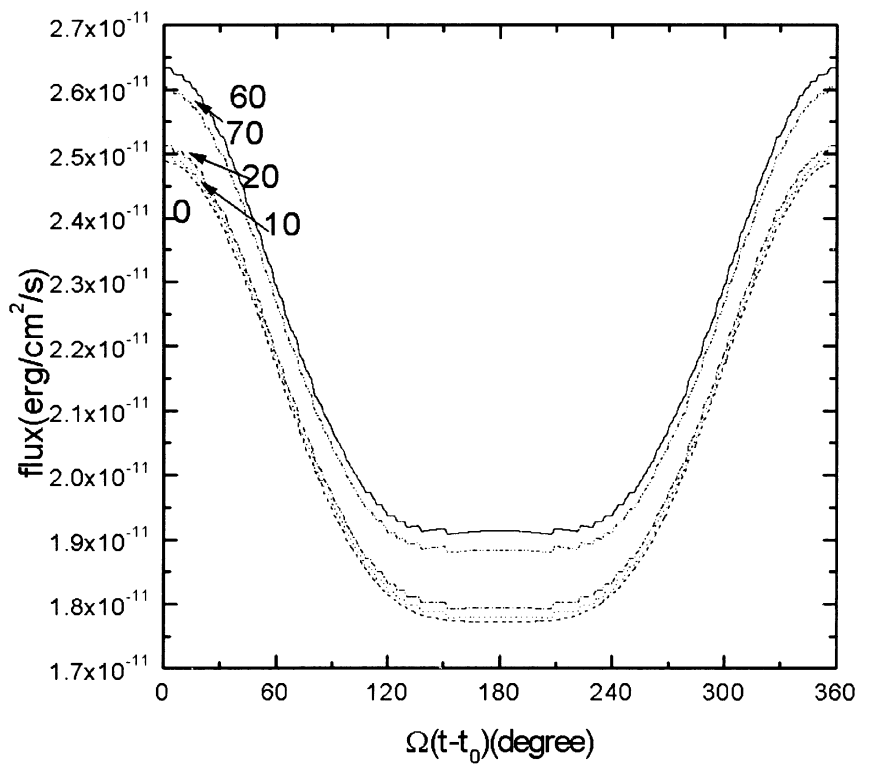

FIg. 18. - Same as Fig. 17, but for $T_{c}=10^{8} \mathrm{~K}$

We define a quantity

$$
E_{\gamma}=\int_{0}^{2 t_{\max }}\left[L(t)-L_{\mathbf{B G}}\right] d t,
$$

where $t_{\max }$ is the time at which $L(t)$ reaches its peak value after the glitch and $L_{\mathbf{B G}}$ is the luminosity before the glitch. This parameter indicates how much glitch energy is released as a pulse. From the $E_{\gamma}$ column in Tables 2 and 3, it can be easily seen that the faster the luminosity $(L)$ reaches its peak value, the smaller is the fraction of the glitch energy eventually emitted as photons at the surface within $2 t_{\max }$. We wish to point out that eventually all the glitch energy will be radiated through the surface in a timescale much longer than $t_{\max }$.

\section{CONCLUSION AND DISCUSSION}

We have employed the relativistic heat diffusion equations to calculate the thermal response of a pulsar to a glitch with energy deposited in a ringlike area around the rotational equator in the inner crust, in a spherical shell, and in a small volume. The "ring" case is always the middle one in terms of the response time, the response period, and the intensity of the response. This is not unexpected, as the volume of energy deposition is smaller than the "shell" case but larger than the "spot" case. Moreover, we have calculated the expected light curves for the thermal X-ray regime as observed after a glitch for the "spot" case and the "ring" case. The "shell" case is ignored because we believe that the modulation of the light curves in this case will be too insignificant to be observed. After a glitch, the energy flux emitted from the surface is not uniform. Excess thermal $\mathrm{X}$-rays are emitted at a particular phase. This alters the $\mathrm{X}$-ray pulse shape of the pulsar. These curves can be used to compare with the actual data obtained to put constraints on the true glitch model, as well as the interior structure of a neutron star.

For the prompt afterglow of a glitch to be detectable, the energy released should be enormous so that it should reradiate over a reasonable timescale and the change in inten- 
sity of the radiation can be observed. Thermal emissions for $\Delta E \gtrsim 10^{42}$ ergs lie in the soft X-ray region (Chong \& Cheng 1994). The thermal transients produced by giant glitches in nearby pulsars may be observable by the Chandra $X$-Ray Observatory, XMM, and Astro-E. These three missions have complementary capabilities in measuring soft X-ray pulses from neutron stars.

For observations taken following a glitch that triggers thermal afterglow, one should be able to detect significant changes in the X-ray pulse shape if the energy release is anisotropic. The results will be valuable in refining neutron star equations of state and gaining a better understanding of the physics behind neutron star transient energy releases (Anderson \& Ögelman 1997).

The defect of this method of finding the internal properties of a neutron star is that if the glitch events occur too frequently, it may result in a pileup of the pulses, as well as a long-term variation of the total thermal radiation, which eventually reduces the detectability of the thermal afterglow of the glitch ( $\mathrm{Li}$ 1997). In the calculations in this paper, several assumptions have been made: the interstellar absorption, the magnetospheric effects, and the possibility of other diffusion mechanisms besides conduction in the pulsar have been neglected; the structure of the pulsar is assumed to be inert to the thermal change of the pulsar; the thermal X-rays emitted from the surface are assumed to be blackbody radiation alone. In future study, we shall take these factors into account.

We thank J. S. Heyl, D. Page, and S. Tsuruta for useful comments and discussion and Terry Boyce for a critical reading. This work is partially supported by an RGC grant from the Hong Kong government, an Outstanding Researcher Award, and a Croucher Foundation Senior Research Fellowship.

\section{REFERENCES}

Alpar, M. A., Anderson, P. W., Pines, D., \& Shaham, J. 1984, ApJ, 276, 325

Alpar, M. A., Chau, H. F., Cheng, K. S., \& Pines, D. 1993, ApJ, 409, 345

Alpar, M. A., Cheng, K. S., \& Pines, D. 1989, ApJ, 346, 823

Alpar, M. A., Langer, S. A., \& Sauls, J. A. 1984, ApJ, 282, 533

Anderson, D. R., \& Ögelman, H. 1997, ApJ, 475, 300

Anderson, P. 1975, preprint (Univ. Cambridge Theor. Condensed Matter No. 1975/5)

Baym, G., Pethick, C., \& Sutherland, P. 1971, ApJ, 170, 299

Baym, G., \& Pines, D. 1971, Ann. Phys., 66, 816

Bildsten, L., \& Epstein, R. I. 1989, ApJ, 342, 951

Cheng, K. S., Alpar, M. A., Pines, D., \& Shaham, J. 1988, ApJ, 330, 835

Cheng, K. S., Chau, W. Y., Zhang, J. L., \& Chau, H. F. 1992, ApJ, 396, 135

Cheng, K. S., \& Li, Y. 1997, in ASP Conf. Proc. 138, Pacific Rim Conference on Stellar Astrophysics, ed. K. L. Chan, K. S. Cheng, \& H. P. Singh (San Francisco: ASP), 195

Cheng, K. S., Li, Y., \& Suen, W. M. 1998, ApJ, 499, L45

Chong, N., \& Cheng, K. S. 1994, ApJ, 425, 210

Epstein, R. I., \& Baym, G. 1992, ApJ, 387, 276

Epstein, R. I., Van Riper, K. A., \& Link, B. 1992, Nature, 359, 616

Flowers, E. G., \& Itoh, N. 1976, ApJ, 206, 218 . 1979, ApJ, 230, 847

Friedman, B., \& Pandharipande, V. R. 1981, Nucl. Phys. A, 361, 502

Greenstein, G., \& Hartke, G. J. 1983, ApJ, 271, 283

Gudmundsson, E. H., Pethick, C. J., \& Epstein, R. I. 1983, ApJ, 272, 286

Harding, A. K., \& Muslimov, A. G. 1998, ApJ, 500, 862

Hernquist, L. 1984, ApJS, 56, 325

Heyl, J., \& Hernquist, L. 1998, MNRAS, 300, 599 1999, MNRAS, 304, L37

Hirano, S., Shibazaki, N., Umeda, H., \& Nomoto, K. 1997, ApJ, 491, 286

Itoh, N., Kohyama, Y., Matsumoto, N., \& Seki, M. 1984, ApJ, 285, 758
Itoh, N., Mitake, S., Iyetomi, H., \& Ichimaru, S. 1983, ApJ, 273, 774

Jones, P. B. 1998, MNRAS, 296, 217

Largaris, I. E., \& Pandharipande, V. R. 1981, Nucl. Phys. A, 359, 349

Lattimer, J. M., Pethick, C. J., Ravenhall, D. G., \& Lamb, D. Q. 1985, Nucl. Phys. A, 432, 646

Li, Y. 1997, M. Phil. thesis, Univ. Hong Kong

Link, B., \& Epstein, R. I. 1991, ApJ, 373, 592

.1996, ApJ, 457, 844

Link, B., Franco, L. M., \& Epstein, R. I. 1998, ApJ, 508, 838

Page, D. 1995, ApJ, 442, 273

Pandharipande, V. R., Pines, D., \& Smith, R. A. 1976, ApJ, 208, 550

Pechenick, K. R., Ftaclas, C., \& Cohen, J. M. 1983, ApJ, 274, 846

Ruderman, M. 1969, Nature, 223, 597 . 1991a, ApJ, 382, 576 .1991b, ApJ, 382, 587

Schaaf, M. E. 1987, Ph.D. thesis, Ludvig-Maximilianf Univ. München 1988, A\&A, 205, 335 1990, A\&A, 235, 499

Smolukowski, R. 1970, Phys. Rev. Lett., 24, 923

Srinivasan, G., Bhattacharya, D., Muslimov, A., \& Tsygan, A. 1990, Curr. Sci., 59,31

Tang, A. P. S. 1999, M. Phil. thesis, Univ. Hong Kong

Thompson, C., \& Duncan, R. C. 1996, ApJ, 473, 322

Tsuruta, S. 1986, Comments Astrophys., 11, 151 1998, Phys. Rep., 292, 1

Van Riper, K. A., Epstein, R. I., \& Miller, G. S. 1991, ApJ, 381, L47

Wiringa, R. B., Fiks, V., \& Fabrocini, A. 1988, Phys. Rev. C, 38, 1010

Yakovlev, D. G. 1982, AZh, 59, 683

Yakovlev, D. G., \& Urpin, V. A. 1980, Soviet Astron., 24, 303 